\title{
Origin and scaling of chaos in weakly coupled phase oscillators
}

\author{
Mallory Carlu, Francesco Ginelli, and Antonio Politi \\ SUPA, Institute for Complex Systems and Mathematical Biology, King's College, University of Aberdeen, \\ Aberdeen AB24 3UE, United Kingdom
}

(Received 11 October 2017; published 10 January 2018)

\begin{abstract}
We discuss the behavior of the largest Lyapunov exponent $\lambda$ in the incoherent phase of large ensembles of heterogeneous, globally coupled, phase oscillators. We show that the scaling with the system size $N$ depends on the details of the spacing distribution of the oscillator frequencies. For sufficiently regular distributions $\lambda \sim$ $1 / N$, while for strong fluctuations of the frequency spacing $\lambda \sim \ln N / N$ (the standard setup of independent identically distributed variables belongs to the latter class). In spite of the coupling being small for large $N$, the development of a rigorous perturbative theory is not obvious. In fact, our analysis relies on a combination of various types of numerical simulations together with approximate analytical arguments, based on a suitable stochastic approximation for the tangent space evolution. In fact, the very reason for $\lambda$ being strictly larger than zero is the presence of finite-size fluctuations. We trace back the origin of the logarithmic correction to a weak synchronization between tangent and phase-space dynamics.
\end{abstract}

DOI: 10.1103/PhysRevE.97.012203

\section{INTRODUCTION}

Oscillatory phenomena are ubiquitous in nature. Collections of interacting oscillators have been widely employed to model a large number of phenomena, ranging from biological [1-3] to social [4] and chemical [5,6] oscillators. Physical examples include lasers [7] and arrays of nonidentical Josephson junctions [8]. While dealing with oscillator ensembles, much attention has been of course devoted to the phenomenon of collective synchronization [9], in which a (large) system of heterogeneous oscillators spontaneously locks at a common frequency above some critical coupling strength [10].

In the weak-coupling limit the oscillators are characterized by a single variable, the phase, while the mutual interaction is mediated by a coupling function which depends only on mutual phase differences (this is the so-called Kuramoto-Daido model [11]). As a result, an ensemble of $N$ oscillators is characterized by at most $N-1$ effective degrees of freedom.

In the thermodynamic limit $N \rightarrow \infty$, the evolution is regular both below and above the synchronization transition, and the largest Lyapunov exponent (LLE) equal to zero. In the small- $N$ limit, the minimal number of oscillators to observe chaos is 4 . This bound has a simple justification: It is, in fact, well known that it is necessary to have at least three (independent) variables to generate chaos. Less obvious is that while $N=4$ is sufficient in heterogeneous oscillators [12], the same bound is attained for identical oscillators only if the coupling function is not purely sinusoidal [13], i.e., going beyond the Kuramoto model [14-17].

For large but finite $N$, there is clear evidence that the LLE is strictly positive [12], but little is known about the underlying mechanisms, even though one might expect that a perturbative approach can be set in the thermodynamic limit, when the effective coupling becomes increasingly weak. In this paper, with the help of direct numerical simulations and approximate analytical arguments, we discuss the finite-size scaling of the LLE and the origin of chaos in the incoherent phase of globally coupled heterogeneous phase oscillators. More specifically, we focus our attention on the celebrated Kuramoto model, the simplest example of heterogeneous, globally coupled, phase oscillators. Its dynamics reads

$$
\dot{\theta}_{i}=\omega_{i}+\frac{g}{N} \sum_{j=1}^{N} \sin \left(\theta_{j}-\theta_{i}\right),
$$

where $g$ is the global coupling parameter and $\theta_{i}$ the phase of oscillator $i$ (with $i=1,2, \ldots, N$ ), and the $\omega_{i}$ are the quenched natural frequencies, typically drawn out of some distribution $P(\omega)$.

Numerical simulations give compelling evidence that the LLE converges to zero in the thermodynamic limit. However, it may scale in two different ways,

$$
\lambda(N) \sim \frac{\ln N}{N} \quad(\text { class I), }
$$

or

$$
\lambda(N) \sim \frac{1}{N} \quad \text { (class II). }
$$

While we are not able to identify exactly the necessary and sufficient conditions for a distribution to belong to a given class, we can at least safely state that whenever the frequencies are generated independently of each other (irrespective whether the distribution itself is Gaussian, uniform in a finite interval, or other), then the logarithmic correction is always present (class I). Independently generated frequencies are of course the most natural choice for many physical or biological setups. On the contrary, if the finite set of frequency spacings is selected in a regular fashion to follow the "macroscopic" shape $P(\omega)$ down to the tiniest scales, the LLE scales as in (3). This is for instance, the case of strictly equispaced frequencies (as already shown in Ref. [12]), a setup which is sometimes considered to be a typical representative of a uniform distribution to 
avoid the burden of averaging over different realizations of the frequencies.

In practice, our analysis reveals that the value of the LLE depends strongly on tiny differences in the actual values of the frequencies, which disappear when the distribution is coarse grained. The presence of quasidegeneracies (almost identical frequencies) definitely enhances the LLE. In fact, as we see in the next section, this is a major source of differences in the typical values of the LLE. However, the overall scenario is more complex than that; the scaling behavior of the LLE depends also on other details such as the presence of long-range order.

Anyway, the analytical arguments based on a (selfconsistent) stochastic approximation of the chaotic dynamics show that the very existence of chaos is due to fluctuations in the tangent space dynamics. Finally, we also put forward a conjecture that traces back the origin of the logarithmic correction of class I to a sort of weak synchronization between tangent- and phase-space coordinates.

The remainder of this paper is organized as follows. In Sec. II, we briefly review the Kuramoto model and present the outcome of numerical simulations (in the incoherent state) performed for different frequency distributions. In Sec. III, we make use of simplified quasiperiodic and discrete-time approximations to put the conjectured existence of two different universality classes on more solid grounds. Semianalytical arguments, based on a stochastic approximation, are put forward in Sec. IV. Finally, our results are discussed in the concluding section.

\section{THE KURAMOTO MODEL}

Since its introduction more than 40 years ago, the Kuramoto model (1) has attracted a good deal of attention in the scientific literature and it has been studied with a combination of analytical techniques and numerical approaches [18].

In particular, it is well known [18] that for a symmetric and unimodal distribution $P(\omega)$, a phase transition is observed for $g=g_{c} \equiv 2 /[\pi P(\bar{\omega})]$, where $\bar{\omega} \equiv \int \omega P(\omega) d \omega$ is the mean frequency [19]. Below $g_{c}$ all oscillators are effectively uncoupled and their phases uniformly distributed; above $g_{c}$ we are in the presence of a symmetry broken, partially synchronized phase, characterized by a finite (complex) order parameter

$$
R e^{i \psi}=\frac{1}{N} \sum_{j=1}^{N} e^{i \theta_{j}} .
$$

In the partially synchronized phase, a finite fraction of oscillators lock at the mean frequency $\bar{\omega}$. They are those whose natural frequency lies closer to the mean one, i.e., in the most densely distributed region for a symmetric unimodal distribution.

The transition is different for nonunimodal distributions. In particular, for the degenerate case of a uniform $P(\omega)$ on a compact interval, one has a first-order transition between a standard incoherent phase and a fully synchronous state, where all oscillators are locked to the same linearly stable periodic orbit with $\dot{\theta}_{i}=\bar{\omega}[20,21]$.

Finite systems are notoriously harder to analyze. Advances have been made concerning, for instance, corrections to the thermodynamic critical coupling parameter value [22], and in the analysis of the dynamical fluctuations of the order parameter [23]. As mentioned in the previous section, numerical studies of large but finite systems are particularly challenging, due to the quenched noise: The results of simulations, in order to be representative of a specific size, have to be averaged over many different realizations of the natural frequencies drawn out of the selected distribution $P(\omega)$. This can be particularly demanding if the observable of interest decreases with the system size (at it is the case of the LLE) and keeps being affected by strong sample-to-sample fluctuations.

In order to get rid of the problem of averaging, some researchers choose to study a single realization of $P(\omega)$, under the assumption that the microscopic details do not alter the scaling behavior. The selection is typically made by choosing the single frequencies so that the corresponding cumulative probability densities are equispaced (see the following subsection for a precise definition). We are going to follow also this approach in the case of both uniform and Gaussian distributions. This strategy greatly reduces the computational burden, but, as already anticipated, we find that the LLE scales differently in these regular cases. In the attempt of clarifying the origin of the different scaling, we introduce and analyze further setups with different degree of (long-range) order and different distributions of the spacing between consecutive frequencies $\omega_{j}$. We will see that these ingredients contribute not only to the quantitative value of the LLE but also to its scaling behavior with $N$.

It is often instructive to rewrite the Kuramoto model to make explicit the mean-field nature of the global coupling. Making use of the order parameter expression (4), one can immediately rewrite the Kuramoto dynamics (1) as

$$
\dot{\theta}_{i}=\omega_{i}+g R \sin \left(\psi-\theta_{i}\right)
$$

revealing that the dynamics of each oscillator is ruled by its phase difference with the average phase $\psi$ and by the interaction strength $g R$.

In the thermodynamic limit, the order parameter $R e^{i \psi}$ settles to either a fixed point $R=0$ or to a limit cycle (a uniform rotation at the average frequency $\bar{\omega}$ ) [24]. As a consequence, the dynamics of the individual phase oscillators decouple and the LLE has to be strictly zero (the opposite claims contained in Ref. [25] are probably due to errors in the numerical simulations). A finite LLE can be observed only in conservative models such as the Hamiltonian mean field, because of subtle correlations induced by the presence of unstable stationary states (see Ref. [26]) In finite systems, however, the order parameter is characterized by fluctuations. In the incoherent regime, for instance [23], $R \sim \frac{1}{\sqrt{N}}$. These fluctuations are the ultimate source of a strictly positive LLE [27].

\section{A. The different frequency setups}

The very same distribution $P(\omega)$ can be generated in different ways. One can see this in the following way. On the one hand, let us order the frequencies $\omega_{i}$ from the smallest to the largest one to produce $y \equiv i / N$ versus $\omega_{i}$. On the other hand, consider the cumulative distribution function

$$
\Phi(\omega) \equiv \int_{-\infty}^{\omega_{j}} P(\omega) d \omega .
$$


So long as in the large- $N$ limit, $y\left(\omega_{i}\right)$ converges to $\Phi(\omega)$, one can claim that the $N$ frequencies $\omega_{i}$ are distributed according to $P(\omega)$. However, for finite $N$, different choices can be made, leading to different finite-size setups. In the following, we consider various choices. Here we introduce and analyse the most natural disordered and regular setups; further options are introduced and discussed in the next section.

Disordered, Gaussian (DG) distributed frequencies: The frequencies are distributed independently of each other and drawn from a normal distribution-perhaps the most popular choice —-with zero average and unit standard deviation,

$$
P_{G}(\omega)=\frac{1}{\sqrt{2 \pi}} e^{-\frac{\omega^{2}}{2}} .
$$

Some preliminary studies can be found in Ref. [28], where a decay slower than $1 / N$ is reported.

Regular, Gaussian $(R G)$ set of frequencies: Given a generic distribution $P(\omega), N$ frequencies $\left\{\omega_{j}\right\}(j=1,2, \ldots, N)$ are generated as follows:

$$
\frac{j-0.5}{N}=\Phi\left(\omega_{j}\right),
$$

where the $1 /(2 N)$ shift is introduced to make the definition more symmetric. In the case of a Gaussian distribution (7), $\operatorname{erf}(z)=2 \Phi(z \sqrt{2})-1$, where erf is the error function. In practice, the frequencies $\omega_{j}$ are determined by applying a Newton's method to find the zeros of

$$
h\left(\omega_{j}\right) \equiv \frac{j-0.5}{N}-\frac{1}{2}\left[1+\operatorname{erf}\left(\frac{\omega}{\sqrt{2}}\right)\right] .
$$

This distribution minimizes the fluctuations of the frequency spacing. It has been already used to study critical properties of the Kuramoto model [29]. In the thermodynamic limit, the synchronization transition takes place at $g_{c}=\sqrt{8 / \pi}$ for both DG and RG sets of frequencies [18].

Disordered, uniformly (DU) distributed frequencies: As for DG, the frequencies are uniformly distributed id variables,

$$
P(\omega)=\left\{\begin{array}{cc}
1 & \text { for } \omega \in[-1 / 2,1 / 2] \\
0 & \text { otherwise }
\end{array} .\right.
$$

As already noted, this setup shows a first-order phase transition from an incoherent to a fully phase-locked phase at $g_{t}=2 / \pi$.

Regular, equispaced (RE) set of frequencies: Applying Eq. (8) with the uniform distribution yields a set of equally spaced frequencies,

$$
\omega_{j}=\frac{2 j-1}{2 N}-\frac{1}{2} .
$$

This setup has been repeatedly investigated [12,21,30] as a testing ground for the properties of the Kuramoto model.

Before proceeding, we remark that, in the following, without loss of generality, we will work in a uniformly rotating frame so that $\bar{\omega}=0$ and the natural frequency distribution $P(\omega)$ is symmetric around zero. Frequencies will be ordered from the smallest to largest one.

In the disordered setups we expect the LLE $\lambda_{\alpha}$ to depend on the realization $\alpha$ of the stochastic process, so that it is necessary to average over a sufficiently large set of realizations. The resulting ensemble average

$$
\lambda=\left\langle\lambda_{\alpha}\right\rangle_{\Omega}
$$

is our first object of investigation, where $\Omega$ denotes the cardinality of the set. In the regular setups no averaging is obviously required.

\section{B. Finite-size scaling of the largest Lyapunov exponent}

We begin our journey by performing a numerical finitesize scaling analysis of the LLE for the Kuramoto model (1). Tangent-space dynamics is ruled by the equation

$$
\dot{\delta} \dot{\theta}_{i}=\frac{g}{N} \sum_{j} \cos \left(\theta_{j}-\theta_{i}\right)\left(\delta \theta_{j}-\delta \theta_{i}\right)=\sum_{j=1}^{N} J_{i j}(\boldsymbol{\theta}) \delta \theta_{j},
$$

where the upper dot denotes the time derivative, while the Jacobian matrix

$$
J_{i j}(\boldsymbol{\theta})=\frac{g}{N}\left[\cos \left(\theta_{j}-\theta_{i}\right)-\delta_{i j} \sum_{k} \cos \left(\theta_{k}-\theta_{i}\right)\right]
$$

is evaluated along the phase-space trajectory $\boldsymbol{\theta}(t) \equiv$ $\left(\theta_{1}, \theta_{2}, \ldots, \theta_{N}\right)$. The symbol $\delta_{i j}$ denotes as usual the Kronecker's delta. In practice, the matrix $J_{i j}$ is the sum of a full and a diagonal matrix such that the sum of all elements along each row is zero. In other words, this is a typical instance of (extended) Laplacian coupling.

It should be noticed that the parameter $g$ cannot be scaled out in spite of it being just a multiplicative factor. In fact, while $g$ can be explicitly removed by rescaling the time variable, the dependence on $g$ would be transferred to the time evolution of the angles $\theta_{i}$. In practice, controlling $g$ is equivalent to controlling the frequency dispersion.

It is convenient to rewrite the evolution equation as

$$
\dot{\delta} \theta_{i}=g\left[-R \cos \left(\psi-\theta_{i}\right) \delta \theta_{i}+Z \cos \left(\beta-\theta_{i}\right)\right],
$$

where $R$ is the Kuramoto order parameter and $\psi$ is its phase, while

$$
Z e^{i \beta}=\frac{1}{N} \sum_{j} \delta \theta_{j} e^{i \theta_{j}}
$$

is the average of the tangent-space variables $\delta \theta_{j}$ oriented according to the corresponding oscillators phases. We shall discuss the role of this term more in detail in Sec. IV.

In the following, we integrate the Eqs. (5) and (15) using a fourth-order Runge-Kutta algorithm, with a time step $\delta t=$ 0.01 . The LLE $\lambda$ measures the asymptotic growth of a generic tangent-space vector $\delta \theta(t) \equiv\left(\delta \theta_{1}, \delta \theta_{2}, \ldots, \delta \theta_{N}\right)$,

$$
\lambda=\lim _{t \rightarrow \infty} \frac{1}{t} \ln \frac{\|\delta \theta(t)\|}{\|\delta \theta(0)\|} .
$$

In practice, the tangent-space vector is rescaled to unit norm every $\Delta t$ time units (unless otherwise stated, we choose $\Delta t=$ 1). This procedure allows us to reconstruct the finite-time Lyapunov exponent (FTLE) as

$$
\lambda_{t}=\frac{\ln \|\delta \theta\|}{\Delta t},
$$

where $\|\delta \theta\|$ is the norm of the tangent vector immediately before rescaling. The LLE of the dynamics is then the asymptotic time average of $\lambda_{t}$.

As anticipated, we concentrate on the incoherent phase. For the Gaussian setup (both disordered and regular frequencies), 
we fix $g=0.4 g_{c}$, while for uniformly distributed frequencies we choose $g=0.4 g_{t}$. This choice is safely far away from the transition point: The correlations among different oscillators that may arise when the coupling $g$ approaches $g_{c}$ or $g_{t}$ are still negligible. However, we have verified that our results hold also for different coupling values (in particular, $g=0.8 g_{c}$, not shown here) in the nonsynchronized regime. Simulations are performed starting from random initial phases and discarding a transient of $T_{0}=N \times 10^{3}$ time units. Ensemble averages in the disordered cases are typically performed over $\Omega=10^{2}$ different frequency realizations, while the single-sample LLE $\lambda_{\alpha}$ is computed over $T=2 N \times 10^{3}$ time units. We have verified that, with this choice, the standard error of the singlesample LLE is at least one order of magnitude smaller than the characteristic sample-to-sample spread of $\lambda_{\alpha}$. Therefore, we can confidently express the numerical uncertainty of our estimate of $\lambda(N)$ with the sample-to-sample standard error,

$$
S_{E}=\frac{\Delta \tilde{\lambda}}{\sqrt{\Omega-1}},
$$

where $\Delta \tilde{\lambda}$ is the standard deviation of the $\lambda_{\alpha} \mathrm{s}$.

In the regular setups, the uncertainty on $\lambda$ is only due to the temporal fluctuations of the FTLE $\lambda_{t}$. In these cases we define the uncertainty as

$$
s_{E}=\frac{\Delta \lambda}{\sqrt{n-1}},
$$

where $\Delta \lambda$ is the standard deviation of $\lambda_{\tau}, \tau$ is a long-enough time to ensure a statistical independence of two consecutive measurement, while $n=T / \tau$ is the number of data points available from a simulation of length $T$ (this latter time is typically chosen on the order of $\sim 10^{6}-10^{7}$ time units). In the remainder of this paper the error bars correspond to one standard error.

The numerical results are resumed in Figs. 1(a) and 1(b), where the scaling of the LLE with $N$ is shown for the abovementioned setups. A common property of all simulations is that $\lambda$ decreases with the system size. This is not surprising since, below threshold, in the thermodynamic limit, the oscillators are mutually uncoupled. A less trivial result is that the regular setups (open black symbols) yield a substantially smaller LLE than the disordered ones (full red symbols). The difference is not only quantitative but even qualitative: A look at Figs. 1(c) and 1 (d) reveals that while the LLE decreases as $1 / N$ in the regular cases, it behaves as

$$
\lambda(N) \sim \frac{\ln N}{N}
$$

in both disordered cases.

It is rather difficult to push the direct numerical simulations of the full model [Eqs. (5) and (15)] beyond $N=10^{4}$. In fact, the increasing demand of CPU time to simulate larger ensembles is accompanied by the need to ensure a constant relative accuracy of the corresponding decreasing LLEs.

In regular setups the problem is worsened by the additional slower decay of the autocorrelation function. In fact, the statistical error, estimated from Eq. (20), increases, due to the increase of the autocorrelation time, which reduces the number $n$ of statistically independent points, within a given time range $T$. The problem is even more pronounced for equispaced (a)

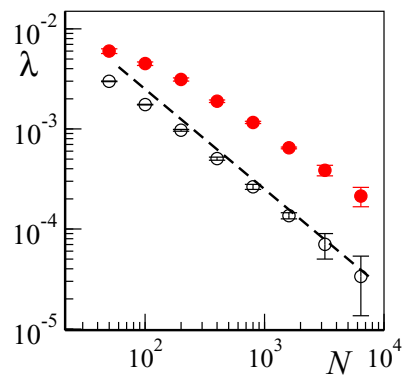

(b)

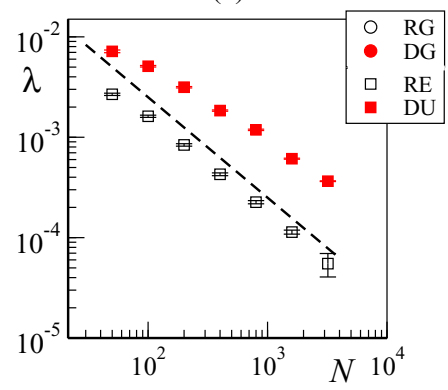

(c)

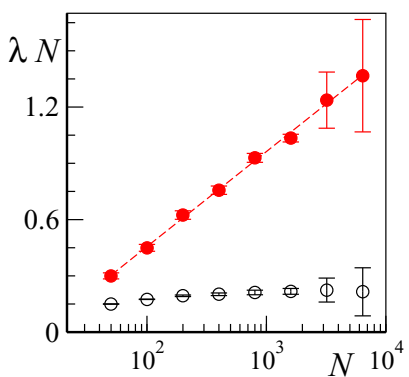

(d)

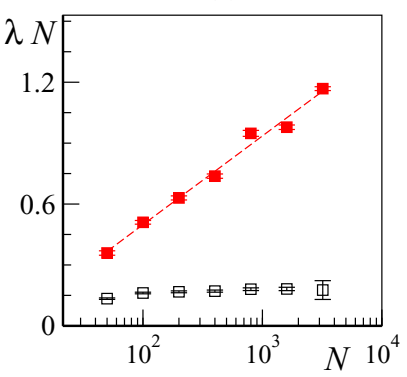

FIG. 1. Finite-size scaling of the largest Lyapunov exponents in the incoherent phase (see text). (a) LLE $\lambda$ vs. system size for disordered Gaussian (DG, full red circles) and regular Gaussian (RG, empty circles) frequencies. (b) Same as (a) but for disordered uniform (DU, full red squares) and regular equispaced (RE, empty squares) frequencies. Axes are in double logarithmic scale, and the black dashed lines mark a decay $\sim 1 / N$. [(c) and (d)] The LLEs of panels (a) and (b) are multiplied by system size $N$ to better show the leading logarithmic correction to the disordered frequencies choices. Axes are now in log-lin scale, and the red dashed lines represent the best logarithmic fit of the disordered frequencies data.

frequencies (11), since in the thermodynamic limit $R=0$ and the dynamics is periodic with a period $T_{P}=2 \pi N$, as is easily seen from Eq. (11). Finite system sizes (and, thereby, nonzero coupling strengths), of course, destroy the exact periodicity, but we have observed that correlations between consecutive periods persist (up to around five periods for our coupling choice) and should be accounted for when estimating $n$. The same effect is also present for regular Gaussian frequencies, although the periodicity is, in this case, approximate even in the zero-coupling case. In fact, on increasing $N$, the frequency distribution is increasingly uniform on microscopic scales. In the central, densest region, expanding the error function in Eq. (9) up to first order in the argument, one finds $T_{P} \approx$ $2 \sqrt{2 \pi} N$.

In disordered setups, these coherence problems are not so crucial, but it is necessary to deal with sample-to-sample fluctuations. Actually, this issue has a conceptual relevance: So long as the relative amplitude of these fluctuations decreases with $N$, one could conclude that the LLE is a well-defined, self-averaging quantity; otherwise, one should conclude that sample-to-sample fluctuations remain relevant in the thermodynamic limit. We have explored this issue by determining the distribution of LLEs for different values of $N$. The results are illustrated in Fig. 2(a), where $\lambda_{\alpha}$ is rescaled to the average value $\left(\Lambda_{\alpha} \equiv \lambda_{\alpha} / \lambda\right)$ to compare the size of the fluctuations 
(a)

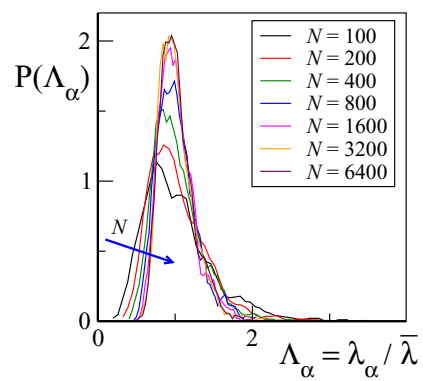

(b)

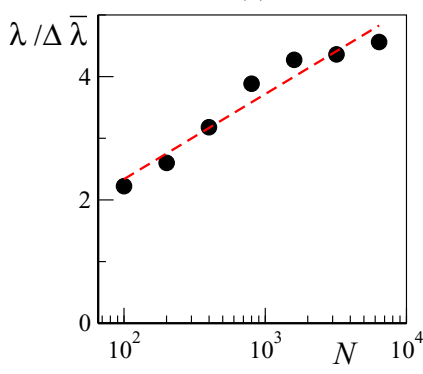

FIG. 2. Marginal self-averaging properties. (a) Probability distributions for the rescaled LLE $\Lambda_{\alpha}$ (see main text) for different system sizes (size is increasing along the blue arrow). Each probability distribution is estimated from $10^{3}$ realizations of the disordered Gaussian frequencies. (b) Inverse ratio $\lambda / \Delta \bar{\lambda}$ vs. system size in a log-lin scale. The dashed red line marks a logarithmic fit.

for different system sizes. The distribution appears to narrow, but the dependence on $N$ is very slow. A more quantitative analysis can be performed by plotting the inverse of the relative standard deviation $\Delta \bar{\lambda} / \lambda$ versus $N$. The data plotted in Fig. 2(b) suggest a logarithmic convergence to zero, i.e., a marginally self-averaging property, which makes large-size accurate simulations rather problematic.

Altogether, we have found numerical evidence that the LLE in the incoherent phase of the Kuramoto model decays to zero as $1 / N$. This result agrees with earlier numerical estimates provided in Ref. [12] for regular equispaced frequencies. On the other hand, our simulations suggest that disordered setups (like the original Kuramoto model) are characterized by a logarithmic correction. As the conjectured existence of two universality classes is only based on numerical simulations, it is desirable to consider as large systems as possible to avoid being misled by uncontrollable finite-size effects. In order to reach larger $N$ values it is necessary to alleviate the simulation burden. This is precisely the goal of the next section.

\section{APPROXIMATE MODELS}

In order to gain insight on the scaling behavior of the LLE discussed in the previous section, here we introduce some approximations and variants of the original model.

\section{A. Quasiperiodic phase-space approximation}

In the thermodynamic limit, i.e., for a strictly infinite $N$, the oscillators are uncoupled and the overall dynamics is quasiperiodic (QP). For a large but finite $N$, the order parameter is small but nonzero: This induces fluctuations around the QP dynamics and modifies the tangent-space dynamics. In order to test to what extent the two effects contribute to the scaling behavior of the LLE, we have decided to investigate a setup where the phase-space dynamics is purely QP,

$$
\dot{\theta}_{i}=\omega_{i} .
$$

Before proceeding in that direction, it is instructive to quantify the effect of the coupling, computing the frequency shift $\Delta \omega_{j} \equiv\left\langle\dot{\theta}_{j}(t)\right\rangle_{t}-\omega_{j}\left(\right.$ here $\langle\cdot\rangle_{t}$ denotes time average). (a)

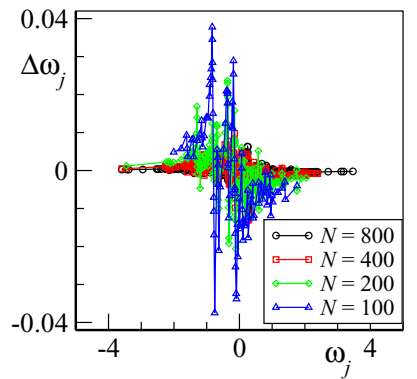

(c)

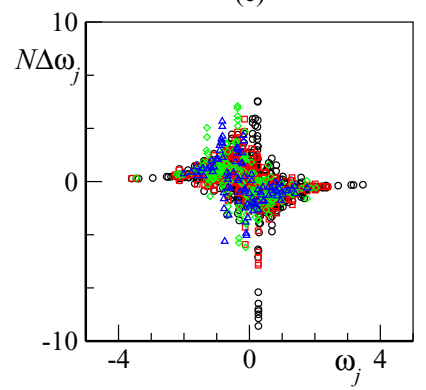

(b)

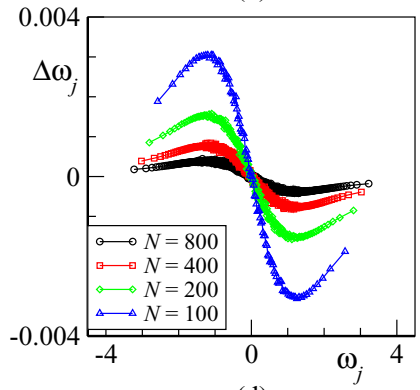

(d)

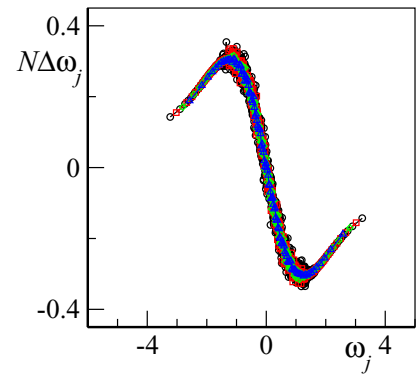

FIG. 3. (a) Frequency shift $\Delta \omega_{j}$ vs. the natural frequency $\omega_{j}$ for different system sizes $N$ for one typical realization of DG frequencies and an average time of $T \approx 10^{6}$ time units. (b) Same as (a) but for RG frequencies. [(c) and (d)] Frequency shift multiplied by the system size $N$ to show the finite-size scaling $1 / N$.

In Fig. 3 we plot $\Delta \omega_{j}$ versus the natural frequency for the two Gaussian setups [DG, panel (a); RG panel (b)]. DG shows larger deviations in the central (denser) part of the frequency range. This actually reflects the tendency of the oscillators to form partially synchronized clusters, where the frequency spacing $\Gamma_{j} \equiv \omega_{j+1}-\omega_{j}$ tends to be smaller (a precursor of the synchronization transition). The RG setup behaves differently: The "response" curve is symmetric (an obvious consequence of the perfect symmetry of the RG distribution) and the frequency shift is much smaller by roughly one order of magnitude. From the overlap in the lower panels, we can appreciate the scaling with $N$ of the frequency shift. The numerics suggests that $\Delta \omega_{j} \sim 1 / N$ in both cases, although the scaling is much cleaner for RG. A possible motivation for the quantitative differences exhibited by the two setups comes from the distribution of the frequency spacings $\Gamma_{j}$. Because of the quenched randomness, in the DG case, $\Gamma_{j}$ can be occasionally very small; in such a case the $j$ th and $j+1$ st oscillators act almost as a single unit within an ensemble of $N / 2$.

The above numerical studies indicate that deviations from the QP zero-coupling behavior are of order $1 / N$. Under the assumption that such deviations do not crucially affect the scaling behavior of the LLE, we focus on the tangent-space evolution ruled by Eq. (15), feeding it with the QP evolution of the single phases. In fact, the results shown in Figs. 4(a) and 4(b), confirm that the regular setup is characterized by a $1 / N$ scaling, while the disordered one exhibits a $(\ln N) / N$ behavior. Additionally, in the disordered case, the LLE is much larger (by about one order of magnitude) in the QP case, with an obvious numerical advantage. 
(a)
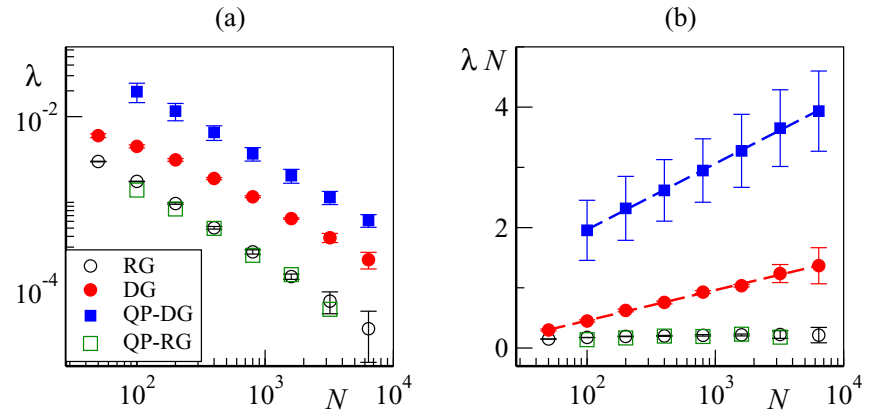

FIG. 4. Quasiperiodic approximation (a) LLEs vs. system size for the quasiperiodic (squares) and full dynamics (circles) in the disordered (full symbols, data averaged over 100 different frequency realizations) and regular (open symbols) Gaussian frequencies setups. Axes are in a doubly logarithmic scale. (b) The LLE of panel (a) are multiplied by system size $N$ to highlight the leading logarithmic correction to the disordered frequencies setups. Axes are now in log-lin scale, and the red dashed line represents the best logarithmic fit of the disordered frequencies data.

\section{B. Discrete-time approximation}

While the quasiperiodic approximation has the advantage of letting us dealing with larger LLEs, thus reducing the statistical fluctuations, it must be noted that it does not speed up significatively the numerics, as it does not free us from the burden of integrating continuous-time differential equations. Under the conjecture that the logarithmic correction is a universal property of a large class of disordered, coupled phase-oscillators, we have further simplified the model. In practice, we have considered discrete-time setups, which are significantly faster to simulate (by at least two orders of magnitude) and thereby allow for more accurate numerical tests. The tangent-space evolution is obtained by discretizing the Kuramoto model, again under the assumption of a quasiperiodic dynamics in real space, $\theta_{i}=\alpha_{i}+\omega_{i} t$,

$$
\begin{aligned}
\delta \theta_{i}(t+1)= & {\left[1-g R(t) \cos \left(\psi-\omega_{i} t-\alpha_{i}\right)\right] u_{i} } \\
& +g Z(t) \cos \left(\beta-\omega_{i} t-\alpha_{i}\right) .
\end{aligned}
$$

By assuming a disordered uniform distribution of frequencies in the interval $[-1 / 2,1 / 2]$ and a uniform distribution of the initial phases $\alpha_{i}$, together with setting $g=1 / 2$ (we have separately verified that the dynamics stays incoherent for this coupling), we have been able to extend the numerical evidence of the class II behavior to much larger system sizes, as it can be appreciated in Fig. 5 (red dots). We are therefore more confident in conjecturing that the logarithmic correction survives in the thermodynamic limit.

Given the opportunity to perform large-size simulations offered by the discrete-time setup, we have decided to explore how large the universality class characterized by the presence of logarithmic corrections is. We have already seen that in regular distributions the LLE scales simply as $1 / N$. In the case of uniform distributions, it is not advisable to investigate equispaced frequencies in the QP approximation, as this would result in a strictly periodic phase-space dynamics that is obviously nongeneric.

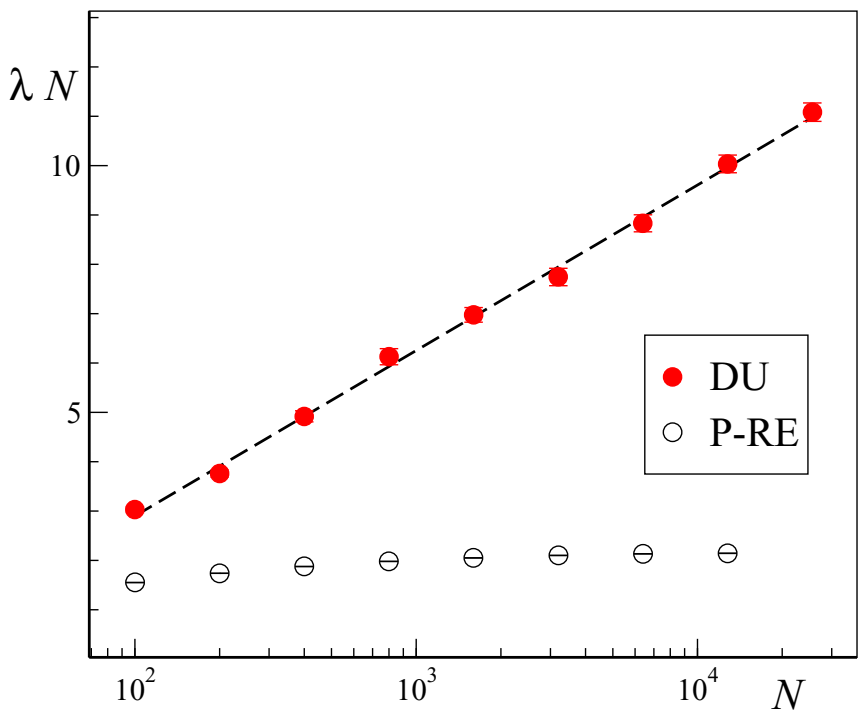

FIG. 5. Rescaled largest Lyapunov exponent for the discrete Kuramoto model vs. system size. Disordered uniformly distributed (DU) frequencies are marked by full red circles, while empty black squares refer to perturbed $(p=1)$ regular equispaced frequencies. Axes are in lin-log scale to highlight the logarithmic correction to the disordered ensemble (black dashed line fit). Data have been averaged over 100 different realizations.

This pathological property has suggested us to consider a variant of the regular distribution, where a quenched microscopic randomness is included,

$$
\omega_{j}=\frac{2 j-1+p \zeta_{j}}{2 N}-\frac{1}{2},
$$

where the $\zeta_{j}$ 's are independent and identically distributed random variables distributed in $[-1,1]$, while $p$ quantifies the strength of the perturbation. In the following we refer to this setup as perturbed regular equispaced (P-RE). For $p=0$, the distribution is perfectly regular. For $0<p<1$ the frequency spacing $\Gamma_{j}$ is strictly larger than $(1-p) / N$; this can be interpreted as the minimal separation between consecutive frequencies. For $p=1$ such a gap vanishes.

Numerical simulations of the P-RE setup (23) reveal that the ensemble averaged LLE $\lambda$ scales as $1 / N$ (see the black squares in Fig. 5 for the case $p=1$ ). Therefore, we can conclude that the presence of stochastic fluctuations is not a sufficient condition to observe the logarithmic correction.

It should, however, be noticed that the P-RE distribution differs from the DU one in the same way that the configuration of a one-dimensional solid differs from a one-dimensional gas (interpreting the frequency values as the positions of "atoms" on a line): Only the former is characterized by long-range order. This leads to conjecture that the absence of long-range order might be a key property.

\section{Frequency spacing distributions}

So far, we can conjecture that long-range order in the frequency distribution suppresses the logarithmic correction to the scaling of the LLE. Can we conclude that the absence of long-range order is a sufficient condition for the scaling $\lambda \sim \ln N / N$ ? 
(a)

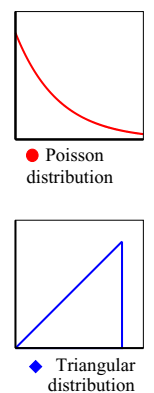

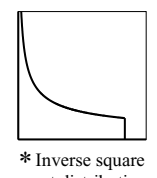
root distribution

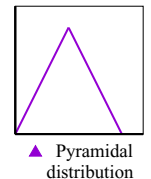

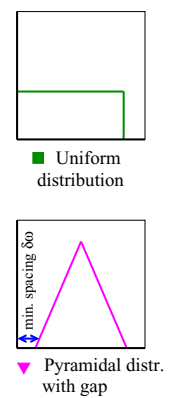

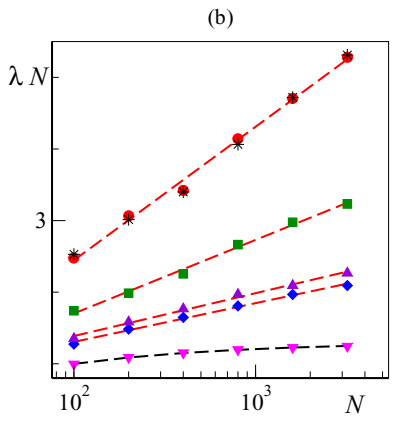

FIG. 6. Quasiperiodic Kuramoto model with disordered frequencies ensembles generated via the frequency spacing distribution method (see text). (a) Graphical depiction of the different frequency spacing distribution tested here. (b) Rescaled largest Lyapunov exponent. The dashed lines have been obtained as the best fit of the numerical data (see text). Data have been averaged over 100 different realizations.

In order to shed light on this issue, we now introduce and analyze further classes of frequencies ensembles. As a matter of fact, instead of generating directly the $N$ frequencies, we now choose to generate directly the $N-1$ frequency spacings $\Gamma_{j}=\omega_{j+1}-\omega_{j}$ from a given probabilty distribution $\Pi(\Gamma)$. The frequencies are afterwards shifted and scaled in order to fill the $[-1 / 2,1 / 2]$ interval.

The selected distributions are graphically depicted in Fig. 6(a), while their mathematical expression can be found in Appendix A. They have been chosen with the goal of clarifying the role of quasidegeneracies by varying the density close to zero. So, we go from (i) the inverse square root, characterized by a divergence in zero, to (ii) the Poisson (which, incidentally, corresponds to the original DU) and (iii) flat distributions, characterized by a finite density in zero, and to (iv) the linearly vanishing density of the triangular and pyramidal distributions. Finally, we have also included a modified pyramidal density with a minimum frequency spacing $b$. It should be noticed that by construction no long-range order is ever present.

Our results, reported in Fig. 6(b) for the quasiperiodic dynamics and tangent space coupling $g=0.4$, indicate that on decreasing the amount of quasidegeneracies (i.e., the spacing probability $\Pi$ near $\Gamma=0$ ), the LLE tends to become smaller, confirming the heuristic idea that they contribute to increasing the degree of instability. However, the overall picture is not as simple as one would like: In all cases the logarithmic correction is present (the best fits for $\lambda N$ are logarithmic in $N$ ), with the exception of the gapped pyramidal case, where our best fit gives $\lambda N=\Lambda_{0}+b / \sqrt{N}$. In other words, only when an identically zero-density appears does the quantitative decrease of the LLE transform into a qualitative change of the scaling behavior. Therefore, we see that the lack of long-range order alone is not sufficient to induce the logarithmic correction: The presence of finite gaps in the frequency spacing seems to suppress the logarithmic correction even in the absence of long-range order.

\section{THEORETICAL CONSIDERATIONS}

In this section we approach the problem from an analytical point of view. We concentrate on the tangent-space dynamics described by Eq. (15), noting that it is determined by two contributions: a multiplicative term $-g R \cos \left(\psi-\theta_{i}\right) \delta \theta_{i}$ and a common pseudoadditive term $g Z \cos \left(\beta-\theta_{i}\right)$ which couples the different tangent-space variables through their weighted average (16). Note that also this latter term is linear in $\delta \theta(t) \equiv$ $\left(\delta \theta_{1}, \delta \theta_{2}, \ldots, \delta \theta_{N}\right)$, so that Eq. (15) is homogeneous in $\delta \theta(t)$, as is expected.

\section{A. Full stochastic approximation}

In the incoherent phase, under the assumption of a stochastic dynamics of both the Kuramoto order parameter $R e^{i \psi}$ and the weighted tangent-vector average $Z e^{i \beta}$, it is possible to derive analytical results, which do not only provide an approximate description of the full model but also help to appreciate the role of the quenched distribution of frequencies in the tangent space evolution.

We start replacing $-g R \cos \left(\psi-\theta_{i}\right)$ and $g Z \cos \left(\beta-\theta_{i}\right)$ in Eq. (15) with two stochastic terms,

$$
\dot{u}_{i}=\xi_{i}(t) u_{i}+\sqrt{\left\langle u^{2}\right\rangle} \eta_{i}(t)
$$

where $\xi_{i}$ and $\eta_{i}$ are independent white noises characterized by variances $\sigma_{\xi}^{2}$ and $\sigma_{\eta}^{2}$, respectively. The square-root term is factored out to show explicitly that the above equation is homogeneous. (For convenience, in the stochastic approximation we denote the tangent vector components $\delta \theta_{i}$ of the original problem as $u_{i}$.)

By further assuming the Stratonovich interpretation for the multiplicative noise, the corresponding Fokker-Planck equation for the probability distribution $\rho(u, t)[31]$ is

$$
\frac{\partial \rho}{\partial t}=-\frac{\partial}{\partial u}\left[\frac{\sigma_{\xi}^{2}}{2} u \rho-\frac{\partial}{\partial u}\left(\frac{\sigma_{\xi}^{2}}{2} u^{2}+\frac{\sigma \eta^{2}}{2}\left\langle u^{2}\right\rangle\right) \rho\right] .
$$

At this level, the presence of a positive Lyapunov exponent $\lambda$ is signalled by an exponential broadening of the distribution $\rho(u, t)$. If we introduce the rescaled variable

$$
v=k u e^{-\lambda t}
$$

then the probability density $S(v) d v=\rho(u, t) d u$ is independent of time. Simple calculations show that

$$
\frac{\partial}{\partial v}\left[\lambda v S-\frac{\sigma_{\xi}^{2}}{2} v S+\frac{\partial}{\partial v}\left(\frac{\sigma_{\xi}^{2}}{2} v^{2}+\frac{\sigma_{\eta}^{2}}{2}\right) S\right]=0,
$$

where we have chosen the normalization constant $k$ so that $\left\langle v^{2}\right\rangle=1$. By imposing the usual no-flux condition, the above equation reduces to

$$
\left(\lambda+\frac{\sigma_{\xi}^{2}}{2}\right) v S+\left(\frac{\sigma_{\xi}^{2}}{2} v^{2}+\frac{\sigma_{\eta}^{2}}{2}\right) \frac{\partial S}{\partial v}=0,
$$

which can be solved, obtaining a sort of generalized Lorentzian shape,

$$
S(v)=\frac{C}{\left(a^{2}+v^{2}\right)^{\tilde{\lambda}+1 / 2}},
$$

where

$$
a=\sigma_{\eta} / \sigma_{\xi} \quad \bar{\lambda}=\lambda / \sigma_{\xi}^{2}
$$


We are left with two undetermined constants, $C$ and $\lambda$. However, they have to satisfy the conditions

$$
\int_{-\infty}^{\infty} S(v) d v=1 \quad \text { and } \quad\left\langle v^{2}\right\rangle=\int_{-\infty}^{\infty} v^{2} S(v) d v=1
$$

On normalizing the area to unity, for finite $a$ one finds that

$$
S(v)=\frac{a^{2 \bar{\lambda}}}{B(1 / 2, \bar{\lambda})\left(a^{2}+v^{2}\right)^{\bar{\lambda}+1 / 2}},
$$

where $B(x, y)$ is the Beta function. Finally, $\bar{\lambda}$ is determined by imposing the self-consistency condition $\left\langle v^{2}\right\rangle=1$,

$$
\frac{B(3 / 2, \bar{\lambda}-1)}{B(1 / 2, \bar{\lambda})} a^{2}=1
$$

By making use of the connection between $B(x, y)$ and $\Gamma(x)$, the above equation reduces to

$$
\bar{\lambda}=1+\frac{a^{2}}{2},
$$

which, in the original framework, becomes

$$
\lambda=\sigma_{\xi}^{2}+\frac{\sigma_{\eta}^{2}}{2} .
$$

Note that this result also holds for $\sigma_{\xi} \rightarrow 0$, i.e., for a vanishing contribution of the diagonal stochastic term. In this case, in fact, Eq. (29) admits the Gaussian solution

$$
S(v)=C e^{-\lambda v^{2} / \sigma_{\eta}^{2}},
$$

which, on imposing the conditions (32), gives $\lambda=\sigma_{\eta}^{2} / 2$.

The opposite limit of a vanishing noisy term $\eta$ is singular. In fact, it is obvious that for $\sigma_{\eta}^{2} \rightarrow 0$, in the absence of the second right-hand side of Eq. (25), the $u$ variables are mutually uncoupled and driven by a zero average term $\xi$, so that the Lyapunov exponent must be zero. This singular behavior is indeed signalled by the solution (30) being nonnormalizable for every value of $\lambda$. We can thus conclude that, at least in our stochastic approximation, chaos emerges from the fluctuations of the weighted tangent-vector average $Z e^{i \beta}$.

It is interesting to compare these results with the stochastic theory developed in Ref. [32] for globally coupled identical chaotic units. In such a case, the maximum Lyapunov exponent is strictly larger than the single-oscillator exponent by an amount equal to the half of the variance of the single-oscillator LE [33]. In the present setup, the fluctuations of the local Lyapunov exponent are represented by $\sigma_{\xi}^{2}$, while $\sigma_{\eta}^{2}$ simulates the fluctuating coupling terms which need to be present to ensure the increase of the LE.

\section{B. Discrete-time version}

Next, we consider the discrete-time equivalent of Eq. (25),

$$
u_{i}(t+1)=\exp \left[\chi_{i}(t)\right] u_{i}(t)+\sqrt{\left\langle u^{2}\right\rangle} \eta_{i}(t)
$$

where $\xi_{i}$ and $\eta_{i}$ are binary variables taking values $\pm \sigma_{\xi}$ and $\pm \sigma_{\eta}$, respectively. This is basically the stochastic version of Eq. (23), with the difference that the term $1+\xi_{i}=1-g R \cos \left(\psi-\theta_{i}\right)$ is replaced by $\exp \left(\chi_{i}\right)$. In the limit $\chi, \xi \ll 1$ the two expressions are similar $(\langle\xi\rangle=0)$; however, in a discrete-time setup, the

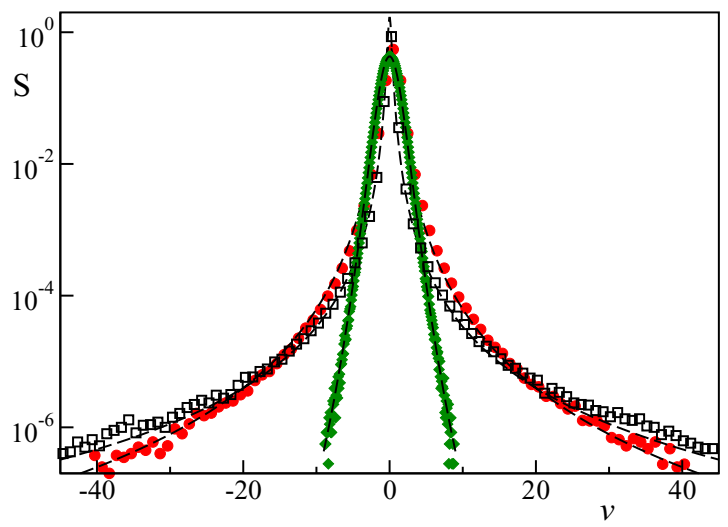

FIG. 7. Discrete-time stochastic approximation. Numerical stationary probability distribution $S(v)$ (symbols) compared with the theory (dashed full lines). Simulations are performed by iterating a system of $4 \times 10^{7}$ elements with $\sigma_{\chi}^{2}=10^{2} f$ and $\sigma_{\eta}^{2}=10^{2}(1-f)$ for $f$ such as to have $a=3$ (green diamonds), $a=1$ (red circles), and $a=0.3$ (black squares).

former one gives a nonzero contribution to the Lyapunov exponent, as seen by expanding the logarithm,

$$
\delta \lambda=\langle\ln (1+\xi)\rangle \approx-\frac{\left\langle\xi^{2}\right\rangle}{2} .
$$

In order to avoid the presence of such a spurious contribution, we prefer to adopt the exponential formulation. We perform numerical simulations of (37), comparing the stationary probability densities $S(v)$ with the Fokker-Planck solution (33). Parametrizing variances as $\sigma_{\chi}^{2}=10^{2} f$ and $\sigma_{\eta}^{2}=10^{2}(1-f)$, from Eqs. (31)-(34) (with the substitution $\xi \rightarrow \chi$ ) we readily obtain $a^{2}=(1-f) / f$ and $\bar{\lambda}=(1+f) /(2 f)$.

The simulations reported in Fig. 7 correspond to three different values of the parameter $a$. The agreement confirms the correctness of the theoretical analysis in a discrete-time setup, too.

\section{Finite-size scaling of fluctuations}

Now we come closer to the Kuramoto-like setup. By comparing Eqs. (15) and (25), we see that $\xi_{i}(t)=-R \cos (\psi-$ $\left.\theta_{i}\right)$, where $R$ is the usual Kuramoto order parameter. In the asynchronous regime, $R$ is well known to be of order $1 / \sqrt{N}$. By neglecting the correlations between $\psi$ and $\theta$, we expect $\sigma_{\xi}^{2} \approx 1 / N$. Analogously, one could also conjecture that $\eta_{i}(t)=\cos \left(\beta-\theta_{i}\right) R / \sqrt{\left\langle u^{2}\right\rangle}$ should be of order $1 / \sqrt{N}$, resulting from the (weighted) average of $N$ terms of order 1. By further assuming a finite decorrelation time, from the theoretical formula (35) one should then conclude that the Lyapunov exponent in a finite incoherent system is expected to be of order $1 / N$.

On the other hand, the simulations reported in the previous sections show that this is not the case for disordered frequency setups, which clearly show a logarithmic correction to the scaling. While there is no reason to challenge the claim that $\left\langle R^{2}(t)\right\rangle \approx 1 / N$, as this is the signature of asynchrony in a finite system, we have monitored $Q_{Z}^{2} \equiv\left\langle Z^{2}\right\rangle$ and $Q_{\beta}^{2} \equiv$ $\left\langle Z^{2} \cos ^{2}\left(\beta-\theta_{i}\right)\right\rangle$ in the discrete Kuramoto model (23) for dif- 
(a)

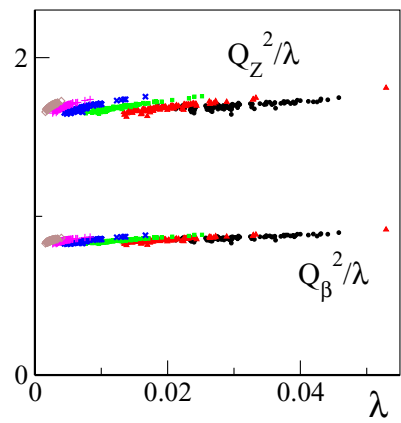

FIG. 8. The two average observables $Q_{Z}^{2} / \lambda$ and $Q_{\beta}^{2} / \lambda$ for different values of $N$ and 100 different realizations of the frequencies in the discrete Kuramoto model (23). (a) Disordered uniform frequency setup. (b) Perturbed regular equispaced frequencies $(p=0.8)$. Single sample time averages are performed over about $4 \times 10^{5}$ time units.

ferent values of $N$ and different realizations of the disordered, uniformly distributed (DU) bare frequencies.

The results are presented in Fig. 8(a), where both $Q_{Z}^{2}$ and $Q_{\beta}^{2}$ are plotted versus the single realization LLE $\lambda_{\alpha}$ (after being divided by $\lambda_{\alpha}$ themselves). We notice a strong correlation between $Q_{Z}^{2}$ and $Q_{\beta}^{2}$ with $\lambda_{\alpha}$, confirming our theoretical insight that they are the basic sources for the scaling of the largest Lyapunov exponent. Note also that no significant correlation is found between single-realization LLEs and the mean-squared Kuramoto order parameter $\left\langle R^{2}\right\rangle$ (not shown). More unexpected is that the correlation persists across different values of $N$. The most important point, however, is that the two quasicurves extrapolate to a finite value for $\lambda_{\alpha}=0$ : This means that in the thermodynamic limit (when $\lambda$ vanishes) $Q_{R}^{2}$ and $Q_{\beta}^{2}$ are proportional to $\lambda_{\alpha}$ itself. In other words, as $\lambda \approx \ln N / N$, so are the two $Q^{2}$ variables, which are therefore responsible for the anomalous scaling. Finally, note also that the ratio between the two quantities is close to $1 / 2$, revealing that the orientation of $Z$ is uncorrelated with the local angle in real space.

For comparison, we also measured $Q_{Z}^{2}$ and $Q_{\beta}^{2}$ for a discrete Kuramoto frequency setup that shows a clear $1 / N$ scaling, namely the P-RE setup. Our results, reported in Fig. 8(b), again show a strong correlation of $Q_{Z}^{2}$ and $Q_{\beta}^{2}$ with the single realization LLE. However, due to the much smaller sampleto-sample fluctuations, in this case the data points for different system sizes are well separated, with a limited variability of $\lambda_{\alpha}$ between realizations. This result confirms that the fluctuations of $Z e^{i \beta}$ are the main source of chaoticity also in this different setup. However, the finite extrapolation of the ratios $Q_{Z}^{2} / \lambda_{\alpha}$ and $Q_{\beta} /{ }^{2} \lambda^{\alpha)}$ now indicates that in this case the two $Q^{2}$ variables scale as $1 / N$.

To summarize, we have been able to trace the scaling of the LLE to the behavior of the fluctuations of the weighted tangent vector average $Z e^{i \beta}$. In typical disordered frequency setups one has

$$
\left\langle Z^{2}\right\rangle=\left\langle\left|\frac{1}{N} \sum_{j} \delta \theta_{j} e^{i \theta_{j}}\right|^{2}\right\rangle \sim \frac{\ln N}{N},
$$

while for more regular distributions, characterized by longrange order and/or nonzero minimum frequency spacings, one

(a)
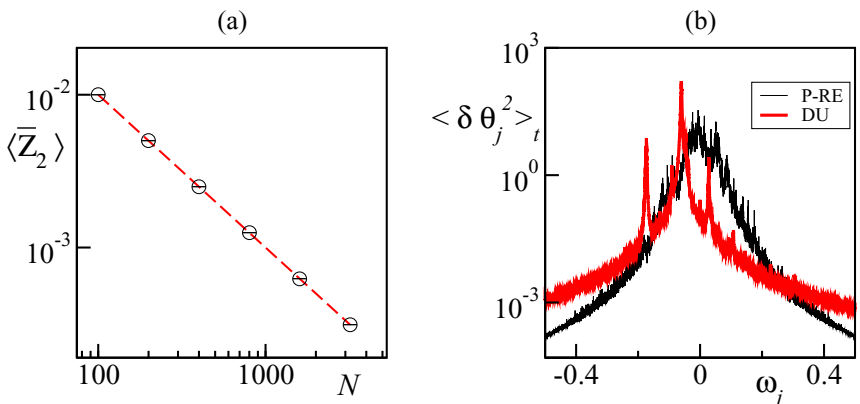

FIG. 9. (a) Finite-size scaling of the reshuffled average $\tilde{Z}^{2}$ for DU frequencies (see text) in the discrete Kuramoto model. Ensemble averages are taken over 100 different realizations of the frequencies. (b) Time-averaged (over $T=4 \times 10^{5}$ time units) square amplitude of the Lyapunov vector components as a function of the corresponding natural frequency for a single frequency realization.

has

$$
\left\langle Z^{2}\right\rangle \sim \frac{1}{N}
$$

\section{Weak synchronization and localization in tangent space}

Finally, we discuss the physical interpretation of the deviations of $\left\langle Z^{2}\right\rangle$ from a $1 / N$ scaling.

To illustrate the point, consider a disordered frequency setup where $\left\langle Z^{2}\right\rangle \sim \ln N / N$ and define the "reshuffled average,"

$$
\tilde{Z}^{2}=\left|\frac{1}{N} \sum_{j} \delta \theta_{j} e^{i \theta_{k}}\right|^{2},
$$

where the $k$ indices are randomly reshuffled versions of the original $j$ indices. It turns out [see Fig. 9(a)] that this reshuffling is sufficient to suppress the logarithmic correction to scaling, i.e., that

$$
\left\langle\tilde{Z}^{2}\right\rangle \sim \frac{1}{N} .
$$

In other words, the logarithmic correction is a manifestation of a form of weak synchronization of the vector $\boldsymbol{\delta} \theta$, i.e., of nontrivial correlations between tangent and phase-space coordinates. This can be also appreciated comparing the average square amplitude of the tangent vector components for disordered (DU) and more regular (P-RE) frequency setups (again in the discrete Kuramoto model). The results are plotted in Fig. 9(b), where we see that the various time-averaged components, plotted as a function of the frequencies, are characterized by substantially different amplitudes for the disordered case. This is a major difference with respect to the regular setup, where all components behave in the same way.

Put in other words, our findings imply that in disordered setups the LLE tangent space vector localizes on a finite subset of frequencies, thus inducing a logarithmic scaling correction in the fluctuations that drive the tangent space dynamics and in the very LLE itself. The presence of long-ranged order and/or of a finite minimum nearest frequency gap, on the other hand, seems to delocalize the tangent space vector, yielding simpler $1 / N$ squared fluctuations in tangent space dynamics and thus suppressing the logarithmic correction in the LLE. 


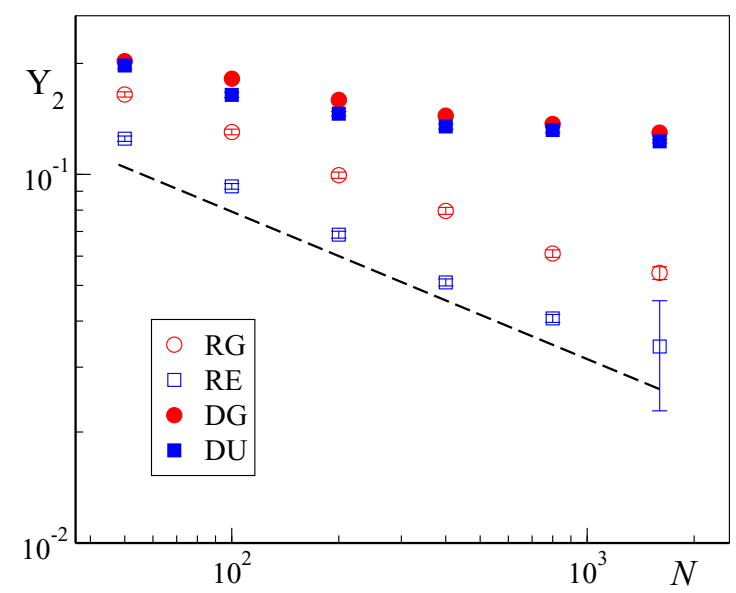

FIG. 10. Finite-size scaling of the tangent vector inverse participation ratio for the full Kuramoto model (1) and different frequency setups: disordered Gaussian (DG, full red circles), disordered uniform (DU, full blue squares), regular Gaussian (RG, empty red circles) and regular equispaced (RE, empty blue squares). Axes are in a double-logarithmic scale. Simulation parameters as in Sec. IIB. The dashed black line marks a power-law decay $N^{-0.4}$.

So far we have developed and tested our theoretical argument under a stochastic approximation (which is indeed rather crude, as it neglects fluctuations correlations) and tested it in discrete-time setups. It is desirable to test a possible link between logarithmic corrections and localization properties of the corresponding Lyapunov vector in the standard Kuramoto model.

Localization properties are captured by the so-called inverse participation ratio (IPR),

$$
Y_{2}=\left\langle\sum_{j} \delta \theta_{j}^{4}\right\rangle_{t}
$$

where vector normalization $\left(\sum_{j} \delta \theta_{j}^{2}=1\right)$ is understood and $\langle\cdot\rangle_{t}$ denotes a time average. Dealing with frequencies ensembles, of course, requires a further average over different realization IPRs, that is, $Y_{2}=\left\langle Y_{2}^{(\alpha)}\right\rangle_{\alpha}$.

In the thermodynamic limit, $Y_{2} \rightarrow 0$ for delocalized vectors (actually, $Y_{2} \sim 1 / N$ for perfectly extended structures). On the other hand, for localized vectors the IPR converges to a finite value, $Y_{2} \rightarrow 1 / \ell$, where $\ell$ is the typical localization length.

We have computed the IPR for the full Kuramoto model (1) and the four frequency setups introduced in Sec. II A. Our results, reported in Fig. 10, unambiguously show that disordered frequency choices (DG and DU) yield a much more localized tangent vector, while regular ones (RG and $\mathrm{RE}$ ) result in a substantially extended structure.

\section{CONCLUSIONS}

We have discussed the way LLE scales to zero in systems of globally coupled oscillators in the asynchronous phase in the context of Kuramoto model. Our analysis shows that the weak chaos in large but finite systems is essentially driven by fluctuations in the tangent-space dynamics. This is yet another instance of a rather general mechanisms first unearthed in globally coupled chaotic units [32], the difference being that there the fluctuations, being an intrinsic property of the single oscillator dynamics, stay finite in the thermodynamic limit.

Our numerical analysis reveals that the scaling of the LLE depends on the way the quenched frequencies are generated on tiny scales but not on the large-scale shape of the distribution. Accordingly, we expect our results to extend to multimodal frequency distributions as well. In the physically meaningful and natural case of independent and identically distributed variables, the LLE scales as $\lambda \sim \ln N / N$, while if the distribution is built using a regular protocol, the scaling is $\lambda \sim 1 / N$ (this is, for instance, the case of equispaced frequencies first found in Ref. [12]). Given such differences, it is legitimate to ask whether the scaling results obtained by using regular protocols are truly universal (see, e.g., Refs. [12,21,30] for the uniform distributions and [29] for the analysis at criticality for a normal distribution).

On a more mathematical, but fundamental, level, it would be desirable to identify the ultimate origin of the logarithmic correction. It is natural to expect that the LLE depends on resonances within suitable $n$-tuples of frequencies. In fact, the Lyapunov-vector structure reported in Fig. 10 indicates a pronounced localization on a special set of frequencies.

This localization is probably the origin of the weak synchronization between tangent- and phase-space dynamics signalled by the logarithmic correction in the scaling of $Z$ (16). However, the main questions are as follows: How are the localization frequencies going to be selected? How many are they? Our simulations revealed the important role of quasidegeneracies: They seem to be a necessary ingredient for the emergence of the logarithmic correction. However, this is clearly not enough as revealed by the two realizations of frequencies, characterized by the same spacing distributions but only one displaying the logarithmic correction. We speculate that long-range order is a second important ingredient, but one cannot exclude that the ultimate answer requires considering subtle number-theoretic properties of the frequency spacings.

We limited ourselves to the analysis of the asynchronous phase. Preliminary numerical simulations suggest that the synchronous phase is characterized by a different scaling. This is not entirely surprising, as the evolution in tangent space is different for two reasons: (i) the Kuramoto order parameter has a finite value and (ii) a finite fraction of oscillators are perfectly locked.

Finally, notice that we have considered only the largest Lyapunov exponent, neglecting the rest of the spectrum. Preliminary results indicate that only a handful of Lyapunov exponents (possibly a nonextensive subset) are positive in finite systems [28]. A careful study of the full Lyapunov spectrum scaling is left for future work.

\section{ACKNOWLEDGMENTS}

A.P. acknowledges D. Pazó for a preliminary analysis. All the authors thank $H$. Chaté for useful discussions. We acknowledge support from EU Marie Curie ITN Grant No. 64256 (COSMOS). 


\section{APPENDIX: FREQUENCY SPACING DISTRIBUTIONS}

For completeness, we report here the analytical expression for the frequency spacing distributions $\Pi(\Gamma)$ studied in Sec. IIIC.

Poisson distribution,

$$
\Pi(\Gamma)=\frac{1}{\Gamma_{c}} e^{-\Gamma / \Gamma_{c}},
$$

with $\Gamma_{c} \sim 1 / N$ to ensure the correct scaling. This choice is fully equivalent to the DU frequency distribution discussed in the main text.

Inverse square root distribution,

$$
\Pi(\Gamma)=\frac{1}{2 \sqrt{\Gamma \Gamma_{c}}},
$$

with $0<\Gamma<\Gamma_{c}$ and the cutoff $\Gamma_{c} \sim 1 / N$ to ensure the correct scaling.
Uniform distribution,

$$
\Pi(\Gamma)=\left\{\begin{array}{ll}
1 & \text { if }|\Gamma| \leqslant 1 / 2 \\
0 & \text { if }|\Gamma|>1 / 2
\end{array} .\right.
$$

Triangular distribution

$$
\Pi(\Gamma)=\frac{2 \Gamma}{\Gamma_{c}^{2}},
$$

with $0<\Gamma<\Gamma_{c}$ and the cutoff $\Gamma_{c} \sim 1 / N$.

Pyramidal distributions,

$$
\Pi(\Gamma)= \begin{cases}C\left(\Gamma-\frac{1-2 a}{N-1}\right) & \text { if } \frac{1-2 a}{N-1} \leqslant \Gamma \leqslant \frac{1}{N}, \\ C\left(\frac{1+2 a}{N-1}-\Gamma\right) & \text { if } \frac{1}{N}<\Gamma \leqslant \frac{1+2 a}{N-1}\end{cases}
$$

where $C=(N-1)^{2} /(2 a)$. The parameter $a \in[0,1 / 2]$ controls the frequency spacing gap. For $a=1 / 2$ no finite gap is present, while for $a<1 / 2$ a finite minimum gap appears (in the main text, $a=0.4$ for the gapped distribution).
[1] T. J. Walker, Science 166, 891 (1969).

[2] J. Aldridge and E. K. Pye, Nature 259, 670 (1976).

[3] D. C. Michaels, E. P. Matyas, and J. Jalife, Circ. Res. 61, 704 (1987).

[4] Z. Néda, E. Ravasz, T. Vicsek, and Y. Brechet, and A. L. Barabási, Phys. Rev. E 61, 6987 (2000).

[5] M. F. Crowley and I. R. Epstein, J. Phys. Chem. 93, 2496 (1989).

[6] W. Wang, I. Z. Kiss, and J. L. Hudson, Phys. Rev. Lett. 86, 4954 (2001).

[7] S. Yu. Kourtchatov, V. V. Likhanskii, A. P. Napartovich, F. T. Arecchi, and A. Lapucci, Phys. Rev. A 52, 4089 (1995).

[8] K. Wiesenfeld, P. Colet, and S. H. Strogatz, Phys. Rev. Lett. 76, 404 (1996).

[9] A. Pikovsky, J. Kurths, and M. Rosenblum, Synchronization: A Universal Concept in Nonlinear Sciences (Cambridge University Press, Cambridge, 2001).

[10] A. T. Winfree, J. Theoret. Biol. 16, 15 (1967).

[11] H. Daido, Physica D 69, 394 (1993).

[12] O. V. Popovych, Y. L. Maistrenko, and P. A. Tass, Phys. Rev. E 71, 065201 (2005).

[13] P. Ashwin, C. Bick, and O. Burylko, Front. Appl. Math. Stat. 2, 00007 (2016).

[14] Y. Kuramoto, in International Symposium on Mathematical Problems in Theoretical Physics, Lecture Notes in Physics, Vol. 39, edited by H. Arakai (Springer, New York, 1975), p. 420.

[15] Y. Kuramoto, Progr. Theoret. Phys. Suppl. 79, 223 (1984).

[16] Y. Kuramoto, Chemical Oscillations, Waves, and Turbulence (Springer, Berlin, 1984).

[17] H. Sakaguchi and Y. Kuramoto, Progr. Theoret. Phys. 76, 576 (1986).

[18] J. A. Acebrón, L. L. Bonilla, C. J. Pérez Vicente, F. Ritort, and R. Spigle, Rev. Mod. Phys. 77, 137 (2005).
[19] Without loss of generality $\bar{\omega}$ can be always set equal to 0 , as we are free to chose the frequency of the reference frame.

[20] H.-A. Tanaka, A. J. Lichtenberg, and S. Oishi, Physica D 100, 279 (1997).

[21] J. L. van Hemmen and W. F. Wreszinski, J. Stat. Phys. 72, 145 (1993).

[22] B. Ottino-Löffler and S. H. Strogatz, Phys. Rev. E 93, 062220 (2016).

[23] H. Daido, J. Phys. A 20, L629 (1987); Prog. Theor. Phys. 81, 727 (1989).

[24] S. H. Strogatz, Phys. D 143, 1 (2000).

[25] G. Miritello,A. Pluchino, and A. Rapisarda, Eur. Phys. Lett. Europhys. Lett. 85, 10007 (2009).

[26] F. Ginelli, K. A. Takeuchi, H. Chaté, A. Politi, and A. Torcini, Phys. Rev. E 84, 066211 (2011).

[27] Above the synchronization transition, the positive contribution to the LLE comes from the dynamics of the unlocked oscillators, the others contributing to strictly negative Lyapunov exponents.

[28] A. Pikovsky and A. Politi, Lyapunov Exponents: A Tool to Explore Complex Dynamics (Cambridge University Press, Cambridge, 2016).

[29] H. K. Hong, H. Chaté, L.-H. Tang, and H. Park, Phys. Rev. E 92, 022122 (2015).

[30] D. Pazo, Phys. Rev. E 72, 046211 (2005).

[31] H. Z. Risken, The Fokker-Planck Equation (Springer, Berlin, 1989).

[32] K. A. Takeuchi, H. Chaté, F. Ginelli, A. Politi, and A. Torcini, Phys. Rev. Lett 107, 124101 (2011).

[33] In Ref. [32], the diffusion constant is uncharacteristically defined as the variance rather than as half the variance. 